\title{
Rough sets applicable in a topology
}

\author{
Nirmala Rebecca Paul ${ }^{1 *}$
}

\begin{abstract}
The paper derives the condition for the rough closure of a subset to contain an element. The relations between rough closure and rough interior are derived. Rough derived set is defined and theorems based on the derived sets are proved. Another rough topology on two equivalence relations is defined. An algorithm is developed to find the core of an information system. Two examples one from the complete system and one from the incomplete system are discussed to find the core.
\end{abstract}

\section{Keywords}

Rough sets, Rough interior, Rough interior, Rough closure and Core.

\section{AMS Subject Classification}

94D05, 68T37.

${ }^{1}$ Department of Mathematics, Lady Doak College, Madurai-625002, Tamil Nadu, India.

*Corresponding author: ${ }^{1}$ nirmalarebeccapaul@ldc.edu.in;

Article History: Received 21 December 2018; Accepted 11 February 2019

(C)2019 MJM.

\section{Contents}

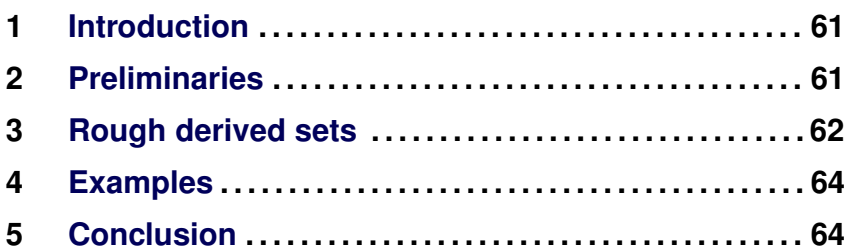

References .................................64

\section{Introduction}

The Rough topology [2] consists of a maximum of three elements. The rough closure is a Kurtowski's closure operator[2]. Some of the properties of rough interior are derived . Rough limit point and the rough derived sets are defined. Theorems involving rough derived set and rough closure are proved. Another rough topology based on two equivalence relations is introduced. An Algorithm is developed to find the CORE of an Information system using the rough topology with respect to two equivalence relations.

\section{Preliminaries}

In this section, we recall some definitions which will be used throughout the paper.

Definition 2.1 (4). Let $U$ be a non-empty finite set of objects called the universe and $R$ be an equivalence relation on $U$ named as the indiscernibility relation. Then $U$ is divided into disjoint equivalence classes. Elements belonging to the same equivalence class are said to be indiscernible with one another. The pair $(U, R)$ is said to be the approximation space. Let $X \subseteq U$.

(i) The lower approximation of $X$ with respect to $R$ is defined and denoted as $L_{R}(X)=\bigcup_{x \in U}\{R(x): R(x) \subseteq X\}$, where $R(x)$ denotes the equivalence class determined by $x$.

(ii) The upper approximation of $X$ with respect to $R$ is defined and denoted as $U_{R}(X)=\bigcup_{x \in U}\{R(x): R(x) \cap X \neq \phi\}$.

(iii) The boundary region of $X$ with respect to $R$ is defined and denoted as $B_{R}(X)=U_{R}(X)-L_{R}(X)$.

Definition 2.2 (1). An information system is of the form $\left(U, A,\left\{V_{a}\right\}, f_{a}\right)$ where $U$ is a non-empty finite set of objects, called the universe, $A$ is a finite non-empty set of attributes, $V_{a}$ is the attribute value set of an attribute $a \in A$ and $f_{a}: U \rightarrow V_{a}$ is called the information function. If $f_{a}(x)$ is equal to a missing value for some $x \in U$ and $a \in A$, then the information system is called an incomplete information system (IIS) Otherwise it is a complete information system(CIS). A missing value is denoted by "*". That is an IIS is of the form $\left(U, A,\left\{V_{a}\right\}, f_{a}\right)$ where $a \in A$ and $* \in \bigcup V_{a}$. An IIS can also be denoted by $(U, A)$.

Definition 2.3 (1). Let $U$ be an universe and $A$ be a finite set of attributes. For any subset $B$ of $A$, there is a binary relation on $U$ corresponding to $B$ given by $R(B)=\{(x, y\} \in U X U$ : 
$f_{a}(x)=f_{a}(y) \operatorname{orf}_{a}(x)=* \operatorname{orf}_{a}(y)=*$ for any $\left.a \in B\right\}$. Then $R(B)$ is a tolerance relation on $U$ (reflexive and symmetric). $S_{B}(x)$ denotes the maximal set of objects which are possibly indiscrenible with $x$ by the tolerance relation on $U$. That is $S_{B}(x)=\{y \in U:(x, y) \in R(B)\}, x \in U$.

Definition 2.4 (1). If $(U, A)$ is an IIS and $B \subseteq A$, then a subset $X$ of $U$ is said to be the tolerance class with respect to $B$, if $(x, y) \in R(B)$ for any $x, y \in X . U / R(B)$ denotes the set of all maximal tolerance classes with respect to $B$ and is called $a$ full cover of $U$.

Definition 2.5 (2). Let $U$ be an universe and $R$ be an equivalence relation on $U$ and $\tau_{R}(X)=\left\{\phi, U, B_{R}(X)\right\}$ where $X \subseteq U$. $\tau_{R}(X)$ satisfies the following conditions

(i) $\phi, U \in \tau_{R}(X)$

(ii)The union of the elements of any sub collection of $\tau_{R}(X)$ is in $\tau_{R}(X)$.

(iii)The intersection of the elements of any finite sub collection of $\tau_{R}(X)$ is in $\tau_{R}(X)$.

$\tau_{R}(X)$ forms a topology on $U$ with respect to the equivalence relation $R .\left(U, \tau_{R}(X)\right)$ is called the rough topological space on $U$ with respect to $X$. The elements of the rough topology are called rough open sets. The complement of the rough open set is called the rough closed set.

Definition $2.6(2)$. If $\left(U, \tau_{R}(X)\right)$ is a rough topological space with respect to $X$, where $X \subseteq U$. Let $A \subseteq U$, then the rough interior of $A$ is defined as the union of all rough open sets contained in $A$ and it is denoted by Rint( $A$ ) which is the largest rough open subset of $A$. The rough closure of $A$ is defined as the intersection of all rough closed sets containing $A$ and it is denoted by $\operatorname{Rcl}(A)$ and it is the smallest rough closed set containing A.

Theorem 2.7 (2). The Rough closure in a rough topological space is the Kuratowski closure operator.

Definition 2.8 (2). In an information system, not all condition attributes depict the decision attribute. The decision depends not on the whole set of condition attributes but on a subset of it is called the CORE.

\section{Rough derived sets}

Remark 3.1. $\left(\tau_{R}(X)\right)^{c}$ also forms a rough topology

In this section some properties of Rough derived sets and rough closure are derived.

Theorem 3.2. In a rough topological space $\left(U, \tau_{R}(X)\right), x \in$ $\operatorname{Rcl}(A)$ if and only if $H \cap A \neq \phi$, for every rough-open set $H$ containing $x$ where $A \subseteq U$.

Proof. If $x \in \operatorname{Rcl}(A)$ and $\mathrm{H}$ is a rough open set containing $\mathrm{x}$, then $\mathrm{U}-\mathrm{H}$ is rough closed. If $A \cap H=\phi$, then $A \subseteq U-H$. That is U-H is a rough closed set containing A. Therefore $\operatorname{Rcl}(A) \subseteq U-H$ Which is a contradiction, since $x \in \operatorname{Rcl}(A)$ but $x \notin U-H$. Hence $A \cap H \neq \phi$ for every rough open set
$\mathrm{H}$ containing $\mathrm{x}$. Conversely if $A \cap H \neq \phi$ for every rough open set $\mathrm{H}$ containing $\mathrm{x}$ and if $x \notin \operatorname{Rcl}(A), x \in U-\operatorname{Rcl}(A)$ which is rough open and hence $(U-\operatorname{Rcl}(A)) \cap A \neq \phi$. But $A \subseteq \operatorname{Rcl}(A)$ and hence $U-\operatorname{Rcl}(A) \subseteq U-A$ which implies $U-\operatorname{Rcl}(A) \cap A \subseteq(U-A) \cap A$ and therefore $(U-A) \cap A \neq \phi$ which is a contradiction. Hence $x \in \operatorname{Rcl}(A)$.

Theorem 3.3. For a rough topological space $\left(U, \tau_{R}(X)\right), A \subseteq$ $U$, the following hold.

(i) $U-\operatorname{Rint}(A)=\operatorname{Rcl}(U-A)$

(ii) $U-\operatorname{Rcl}(A)=\operatorname{Rint}(U-A)$

Proof. i)Let $x \in U-\operatorname{Rint}(A)$. That is $x \notin \operatorname{Rint}(A)$. Therefore $\mathrm{H}$ will not be contained in A for everyery rough open set $\mathrm{H}$ containing x. That is $H \cap U-A \neq \phi$ for every rough open set containing $\mathrm{x}$. Therefore $x \in \operatorname{Rcl}(U-A)$. That is $U-$ $\operatorname{Rint}(A) \subseteq \operatorname{Rcl}(U-A)$. Conversely if $x \in \operatorname{Rcl}(U-A)$ then $H \cap U-A \neq \phi$, for every rough open set $\mathrm{H}$ containing $\mathrm{x}$. That is $x \notin \operatorname{Rint}(A)$. Therefore $x \in U-\operatorname{Rint}(A)$. Thus $\operatorname{Rcl}(U-$ $A) \subseteq U-\operatorname{Rint}(A)$. Therefore U-Rint(A)=Rcl(U-A).

(ii). $A \subseteq \operatorname{Rcl}(A) . U-\operatorname{Rcl}(A) \subseteq U-A$. Since $\mathrm{U}$ - $\operatorname{Rcl}(\mathrm{A})$ is a rough open set set contained in U-A. But $\operatorname{Rint}(\mathrm{U}-\mathrm{A})$ is the largest rough open set contained in U-A. Hence $U-\operatorname{Rcl}(A) \subseteq$ $\operatorname{Rint}(U-A)$. Let $x \in \operatorname{Rint}(U-A)$. But $\operatorname{Rint}(\mathrm{U}-\mathrm{A})$ is disjoint from A. Therefore $\operatorname{Rint}(U-A) \cap A=\phi$. By the theorem $4.1 x \notin \operatorname{Rcl}(A)$. Hence $x \in U-\operatorname{Rcl}(A)$. Thus $\operatorname{Rint}(U-A) \subseteq$ $U-\operatorname{Rcl}(A)$.Hence $\mathrm{U}-\operatorname{Rcl}(\mathrm{A})=\operatorname{Rint}(\mathrm{U}-\mathrm{A})$

Theorem 3.4. For a rough topological space $\left(U, \tau_{R}(X)\right), A \subseteq$ $U$, the following hold (i) $\operatorname{Rint}(A)=U-\operatorname{Rcl}(U-A)($ ii) $\operatorname{Rcl}(A)=U$ $\operatorname{Rint}(U-A)$

Proof. :By taking complements on both sides of (i) and (ii) in the theorem 4.2 $\operatorname{Rint}(\mathrm{A})=\mathrm{U}-\operatorname{Rcl}(\mathrm{U}-\mathrm{A}) \operatorname{Rcl}(\mathrm{A})=\mathrm{U}-\operatorname{Rint}(\mathrm{U}-\mathrm{A})$.

Example 3.5. Let $X=\{a, b, c, d\}$ and $U / R=\{\{a\},\{c\},\{b, d\}\}$. Let $X=\{a, b\}$. Then $\tau_{R}(X)=\{\phi, U,\{b, d\}\}$. The rough closed sets are $\{\phi, U,\{a, c\}\}$. If $A=\{a, c\}$ then $U-\operatorname{Rint}(A)=$ $U$ andRcl $(U-A)=U$. Also $U-\operatorname{Rcl}(A)=\{b, d\}=\operatorname{Rint}(U-$ A)

Theorem 3.6. In a rough topological space $\left(U, \tau_{R}(X)\right)$, for $A, B \subseteq U$ (i) $A$ is rough open if and only if $\operatorname{Rint}(A)=A$ (ii) $\operatorname{Rint}(\phi)=\phi, \operatorname{Rint}(U)=U($ iii $)$ If $A \subseteq B$ then $\operatorname{Rint}(A) \subseteq \operatorname{Rint}(B)$ (iv) $\operatorname{Rint}(A) \cup \operatorname{Rint}(B) \subseteq \operatorname{Rint}(A \cup B)(v) \operatorname{Rint}(A \cap B)=\operatorname{Rint}(A) \cap$ $\operatorname{Rint}(B)(v i) \operatorname{Rint}(\operatorname{Rint}(A))=\operatorname{Rint}(A)$

Proof. (i)A is rough open if and only if U-A is rough closed. $A$ is rough closed if and only if $\operatorname{Rcl}(\mathrm{U}-\mathrm{A})=\mathrm{U}-\mathrm{A}$, if and only if $\mathrm{U}-\operatorname{Rcl}(\mathrm{U}-\mathrm{A})=\mathrm{A}$ if and only if $\operatorname{Rint}(\mathrm{A})=\mathrm{A}$ by the Theorem 4.2 (ii). Since $\phi$ and $\mathrm{U}$ are rough open $\operatorname{Rint}(\phi)=\phi, \operatorname{Rint}(\mathrm{U})=\mathrm{U}$ (iii) $A \subseteq B$ implies $U-B \subseteq U-A$. Therefore $\operatorname{Rcl}(U-B) \subseteq$ $\operatorname{Rcl}(U-A) . U-\operatorname{Rcl}(U-A) \subseteq U-\operatorname{Rcl}(U-B)$. Thus Rint $(A) \subseteq$ $\operatorname{Rint}(B)$

(iv) By (iii) $\operatorname{Rint}(A) \subseteq \operatorname{Rint}(A \cup B), \operatorname{Rint}(B) \subseteq \operatorname{Rint}(A \cup B)$. Hence $\operatorname{Rint}(A) \cup \operatorname{Rint}(B) \subseteq \operatorname{Rint}(A \cup B)$

(v) $\operatorname{Rint}(A \cap B) \subseteq \operatorname{Rint}(A), \operatorname{Rint}(A \cap B) \subseteq \operatorname{Rint}(B) \operatorname{Rint}(A \cap$ 
$B) \subseteq \operatorname{Rint}(A) \cap \operatorname{Rint}(B) . \operatorname{Rint}(A) \subseteq A, \operatorname{Rint}(B) \subseteq B, \operatorname{Rint}(A) \cap$ $\operatorname{Rint}(B) \subseteq A \cap B$. $\operatorname{Rint}(A) \cap \operatorname{Rint}(B)$ is a rough open set contained $A \cap B$. But $\operatorname{Rint}(A \cap B)$ is the largest rough open set contained in $A \cap B$. Hence. $\operatorname{Rint}(A) \cap \operatorname{Rint}(B) \subseteq \operatorname{Rint}(A \cap B)$. (vi)Rint(A) is the rough open set and hence by $(\mathrm{i}) \operatorname{Rint}(\operatorname{Rint}(\mathrm{A}))=\operatorname{Rint}(\mathrm{A})$

Definition 3.7. Let $\left(U, \tau_{R}(X)\right)$ be a rough topological space and $A \subseteq U$. An element $x \in U$ is said to be a rough limit point of $A$ if $G \cap A-\{x\} \neq \phi$ for every rough open set $G$ containing $x$.The set of all rough limit points of $A$ is denoted by $R D(A)$ and is called rough derived set of $A$.

Example 3.8. Let $U=\{a, b, c, d\}, U / R=\{\{a\},\{b\},\{c, d\}\}$ and $X=\{b, d\}, \tau_{R}(X)=\{\phi, U,\{c, d\}\}$. If $A=\{a, c\}$ then $R D(A)=\{a, b, d\}$

Theorem 3.9. $\operatorname{Rcl}(A)=A \cup R D(A)$, where $A$ is a subset of $U$.

Proof. If $x \in A \cup R D(A)$ then $x \in A$ or $x \in R D(A)$. If $x \in A$ then $x \in \operatorname{Rcl}(A)$. Therefore let $x \notin A$, then $x \in R D(A)$. Therefore for every rough open set $\mathrm{G}$ containing x,$G \cap(A-\{x\}) \neq \phi$ - Since x does not belongs to $\mathrm{A}, G \cap A \neq \phi$. Therefore $x \in \operatorname{Rcl}(A)$. Thus $A \cup \operatorname{RD}(A) \subseteq \operatorname{Rcl}(A)$. If $x \in \operatorname{Rcl}(A)$ and $x \in A$ then $x \in A \cup R D(A)$. If $x \in R \operatorname{Rcl}(A)$ but $\mathrm{x}$ does not belong to A, then $G \cap A \neq \phi$, for evry rough open set $\mathrm{G}$ containing $\mathrm{x}$ and hence $G \cap(A-\{x\}) \neq \phi$. Therefore $x \in R D(A)$. That is $x \in A \cup R D(A)$. Thus $\operatorname{Rcl}(A) \subseteq A \cup R D(A)$. Hence $\operatorname{Rcl}(A)=$ $A \cup R D(A)$

Corollary 3.10. A subset of $U$ is rough closed if and only if $R D(A) \subseteq A$.

Proof. A is rough closed if and only if $\operatorname{Rcl}(\mathrm{A})=\mathrm{A}$ if and only if $A \cup R D(A)=A$, if and only if $R D(A) \subseteq A$.

Theorem 3.11. If for any two subsets $A, B$ of $U$ then (i) if $A \subseteq B$ then $R D(A) \subseteq R D(B)$

(ii) $R D(A \cup B)=R \bar{D}(A) \cup R D(B)$

(iii) $R D(A \cap B) \subseteq R D(A) \cap R D(B)$

(iv) $R D(\phi)=\phi$

(v) $x \in R D(A)$ implies $x \in R D(A-\{x\})$

Proof. (i) Let $A \subseteq B$ and $x \in R D(A)$. Then for every rough open set $\mathrm{G}$ containing x, $G \cap(A-\{x\}) \neq \phi$. Since $A \subseteq B, G \cap$ $(B-\{x\}) \neq \phi$ and hence $x \in R D(B)$. Thus $R D(A) \subseteq R D(B)$. (ii) Since $A \subseteq(A \cup B)$ and $B \subseteq(A \cup B), R D(A) \subseteq R D(A \cup$ $B)$ and $R D(B) \subseteq R D(A \cup B)$ and hence $R D(A) \cup R D(B) \subseteq$ $R D(A \cup B)$. Let $x \notin R D(A) \cup R D(B)$. Then $x \notin R D(A)$ and $x \notin$ $R D(B)$. Therefore there exists rough open sets $\mathrm{G}$ and $\mathrm{H}$ containing $x$ such that $G \cap(A-\{x\})=\phi$ and $H \cap(B-\{x\})=\phi$ . Since $(G \cap H) \subseteq G$ and $H,(G \cap H) \cap(A-\{x\})=\phi$ and $(G \cap H) \cap(B-\{x\})=\phi$. Also $G \cap H$ is a rough open containing x. Therefore $(G \cap H) \cap[A \cup B-\{x\}]=\phi$. That is $\mathrm{x}$ is not a rough limit point of $A \cup B$. That is $x \notin R D(A \cup B)$. Thus $x \notin(R D(A) \cup R D(B))$ which implies that $x \notin R D(A \cup B)$. Thus $x \in R D(A \cup B)$ which implies that $x \in(R D(A) \cup R D(B))$.

Thus $R D(A \cup B) \subseteq R D(A) \cup R D(B)$. Hence $R D(A \cup B)=R D(A) \cup$ $R D(B)$.

(iii) Since $A \cap B \subseteq A$ and $A \cap B \subseteq B, R D(A \cap B) \subseteq R D(A), R D(A \cap$ $B) \subseteq R D(B) \cap R D(A)$.

(iv) Let $x \in U$. If $x \in R D(\phi)$, then every rough open set $\mathrm{G}$ containing $\mathrm{x}$ has atleast one point of $\phi$ other than $\mathrm{x}$. But $\phi$ has no point. Therefore, no point of $\mathrm{U}$ can be a limit point of $\mathrm{A}$. That is $R D(\phi)=\phi$

(v) If $x \in R D(A)$ then $G \cap(A-\{x\}) \neq \phi$ for every rough open set $\mathrm{G}$ containing x. Since $A-\{x\}-\{x\}=A-\{x\}, G \cap(A-$ $\{x\}-\{x\} \neq \phi$, for every rough open set $\mathrm{G}$ containing $\mathrm{x}$. Therefore $x \in R D(A-\{x\})$. Thus $R D(A) \subseteq R D(A-\{x\})$

Example 3.12. Equality does not hold in (iii). For example let $U=\{a, b, c, d\}$ with $U / R=\{\{a\},\{b\},\{c, d\}\}$ and let $X=$ $\{a, b\}, A=\{a, b\}, B=\{c, d\} . \tau_{R}(X)=\{\phi, U\} . A \cap B=\phi$. $R D(A \cap B)=\phi . R D(A) \cap R D(B)=U$

Theorem 3.13. If $A$ is a singleton subset of $U$ and $\left(U, \tau_{R}(X)\right)$ is a rough topological space, then $R D(A)=\operatorname{Rcl}(A)-A$

Proof. If $x \in R D(A)$, then for every rough open set $\mathrm{G}$ containing $\mathrm{x}, G \cap(A-\{x\}) \neq \phi$. Then $x \notin A$. Since if $x \in A$, then $A=\{x\}$ and hence $G \cap(A-\{x\})=\phi$. But $R D(A) \subseteq \operatorname{Rcl}(A)$. Thus $x \in \operatorname{Rcl}(A)$ but $x \notin A$. whenever $x \in R D(A)$. Therefore $R D(A) \subseteq \operatorname{Rcl}(A)-A$. If $x \in \operatorname{Rcl}(A)-A$ then $x \in \operatorname{Rcl}(A)$ but $x \notin A$. Therefore $G \cap A \neq \phi$, for every rough open set $\mathrm{G}$ containing x. That is $G \cap(A-\{x\}) \neq \phi$ for every rough open set $\mathrm{G}$ containing $\mathrm{x}$. Therefore $x \in R D(A)$. Thus $\operatorname{Rcl}(A)-$ $A \subseteq R D(A)$.HenceRD $(A)=\operatorname{Rcl}(A)-A$, if $\mathrm{A}$ is a singleton set.

Rough topology with two equivalent relations are defined and a algorithm is developed to find the core of an information system.

Definition 3.14. Let $U$ be an Universe and $R, S$ be any two equivalence relations on $U$ and $X$ is a subset of $U$. The lower approximations corresponding to $R * S$ is defined as $L_{R * S}(X)=$ $\bigcup_{x \in U}\{x: R(x) \subseteq X$ and $S(x) \subseteq X\}$ where $R(x), S(x)$ denote the equivalence class determined by $x$.

The upper approximation of $X$ corresponding to $R * S$ is defined as $U_{R * S}(X)=\bigcup_{x \in U}\{x: R(x) \cap X \neq \phi \operatorname{or} S(x) \cap X \neq \phi\}$

The boundary of $X$ corresponding to $R * S$ is defined as $B_{R * S}(X)=$ $U_{R * S}(X)-L_{R * S}(X)$.

Then $\tau_{R * S}(X)=\left\{\phi, U, B_{R * S}(X)\right\}$ is a rough topology with respect to two equivalence relations.

Example 3.15. Let $X=\{a, b, c, d\} U / R=\{\{a\},\{b, c\},\{d\}\}$ $U / S=\{\{a, b\},\{c\},\{d\}\}$. Let $X=\{a, c\} . L_{R * S}(X)=\phi$, $U_{R * S}(X)=\{a, b, c\}, B_{R * S}(X)=\{a, b, c\} . L_{R}(X)=\{a\}$, $U_{R}(X)=\{a, b, c\}, B_{R}(X)=\{b, c\}$.

$L_{S}(X)=\{c\}, U_{S}(X)=\{a, b, c\}, \tau_{R}(X)=\{\phi, U,\{b, c\}\}, \tau_{S}(X)=$ $\{\phi, U,\{a, b\}\}, \tau_{R * S}(X)=\{\phi, U,\{a, b, c\}\}$

\section{ALGORITHM}


A new algorithm is developed to find the CORE of an information system.

Setp 1. Form the equivalence classes $\mathrm{R}$ with respect to all conditional attributes and $\mathrm{X}$ be a subset of the Universe. Find the rough topology with respect to all condition attributes.

Step 2 Form the equivalence classes Ri by removing one condition attribute from the set of condition attributes. Find the Rough topology with respect to $\mathrm{R} * \mathrm{Ri}$ and the set $\mathrm{Ci}$ by deleting one attribute from $\mathrm{C}$.

Step 3 Repeat step 2 for all condition attributes.

Step 4. Find the set of condition attributes $C_{i}$ which results in $\tau_{R * R_{i}}(X)=\tau_{R}(X)$

Step 5 The intersection of all condition attributes $C_{i}$ from step 4 gives the CORE of an information system.

Let (U,A) be an information system. Here $\mathrm{U}$ is an universe, $\mathrm{A}$ is the set of attributes which is divided into a set of $\mathrm{C}$ of condition attributes and a set of $\mathrm{D}$ of decision attribute. The rows of the table represent the members of the universe and the columns represent the conditional attributes.

\section{Examples}

Example 1.

The following table gives six samples of water taken to test the portability of water. If the sample contains the chemical parameters not within the permissible limit will have an impact which is given by the following table.

\begin{tabular}{|c|c|c|c|c|c|}
\hline Parameters & \multicolumn{5}{|c|}{ Impacts } \\
\hline TDS & \multicolumn{4}{|c|}{ Bitter taste, formation of kidney stone } \\
\hline Nitrate & \multicolumn{4}{|c|}{ Blue baby disease in infant } \\
\hline $\mathrm{pH}$ & \multicolumn{4}{|c|}{ leads to ulcer in the stomach } \\
\hline Flouride & \multicolumn{4}{|c|}{ Bone damage, Fluorosis } \\
\hline \hline Samples & F & N & TDS & pH & usability \\
\hline 1 & yes & no & yes & yes & yes \\
\hline 2 & No & No & yes & yes & No \\
\hline 3 & yes & No & no & yes & No \\
\hline 4 & yes & No & yes & No & No \\
\hline 5 & yes & yes & yes & yes & yes \\
\hline 6 & yes & no & no & no & no \\
\hline
\end{tabular}

Here F represents Flouride, N represents Nitrate,Hard represents hardness respectively. Here "No" represents that the parameter present in the sample is not within the permissible limit and "Yes" represents that the parameter present in the sample is within the permissible limit.

Step 1The equivalence classes corresponding to all conditional attributes are given by and name it as

$R=\{\{1\},\{2\},\{3\},\{4\},\{5\},\{6\}\} . X=\{1,5\} \tau_{R}(X)=\{\phi, U\}$

Step $2 R_{1}=\{\{1,2\},\{3\},\{4,6\},\{5\}\} C_{1}=\{N, T D S, P h\}$

. $\tau_{R * R_{1}}(X)=\{\phi, U,\{1,2\}\}$

Step $3 R_{2}=\{\{1\},\{2\},\{3\},\{4,6\},\{5\}\}, C_{2}=\{F, T D S, P h\}$, $\tau_{R * R_{2}}(X)=\{\phi\}$

$R_{3}=\{\{1,3\},\{2\},\{4,6\},\{5\}\}, C_{3}=\{F, N, P h\}, \tau_{R * R_{3}}(X)=$ $\{\phi, U,\{1,3\}\}$,

$R_{4}=\{\{1,4\},\{3,6\},\{2\},\{5\}\}, C_{4}=\{F, N, T D S\}$
Here the rough topology coincides for the set $\mathrm{C} 2$. Hence the CORE is the set $=\{F, T D S, p H\}$

Example 2

This example gives information about patients with different symptoms of breast cancer namely lump in breast, inverted nipple, rashes, nipple discharge and swelling in the armpit and they are represented shortly by L,I,R,D and S

\begin{tabular}{|c|c|c|c|c|c|c|}
\hline Patients & L & $\mathrm{I}$ & $\mathrm{R}$ & $\mathrm{D}$ & $\mathrm{S}$ & Breast cancer \\
\hline 1 & yes & yes & $*$ & yes & no & yes \\
\hline 2 & yes & yes & yes & $*$ & $*$ & yes \\
\hline 3 & no & yes & no & $*$ & yes & no \\
\hline 4 & yes & no & $*$ & no & $*$ & no \\
\hline 5 & no & yes & $*$ & yes & yes & no \\
\hline 6 & yes & $*$ & no & yes & $*$ & yes \\
\hline
\end{tabular}

The columns of the table represent the symptoms for breast cancer and the rows represent the patients. The entries in the table are attribute values. The given information system is incomplete and is given by (U,A) where $U=\{1,2,3,4,5,6\}$ and $A=\{L, I, R, D, S$, Breastcancer $\}$ which is divided into a set $\mathrm{C}$ of condition attributes given as $C=\{L, I, R, D, S\}$ and the set of decision attribute $D=\{$ Breastcancer $\}$. The attribute I generates the tolerance classes $\{1,2,3,5,6\}$ and $\{4,6\}$. Since the missing attribute value for 6 can be yes or no. The maximal tolerance classes for other combination of attributes can be formed. Considering all condition attributes together the maximal tolerance classes are $\{\{1,2\},\{1,6\},\{3,5\},\{4\}\}$ Step 1. $R=\{\{1,2\},\{1,6\},\{3,5\},\{4\}\}, X=\{1,2,4,6\}$. $L_{R}(X)=\{1,2,4,6\}=U_{R}(X)=\{1,2,4,6\}, B_{R}(X)=\{\phi\}$, $\tau_{R}(X)=\{\phi, U\}$.

Step $2 R_{1}=\{\{1,2\},\{3,5,6\},\{4\},\{2,5\},\{1,6\}\}$ which is obtained by deleting $\mathrm{L}$ from the set of condition attributes. $\tau_{R * R_{1}}(X)=\{\phi, U,\{2,3,5,6\}\}$.

Step $3 R_{2}=\{\{1,2\},\{1,6\},\{2,4\},\{3,5\}\}$ which is obtained by deleting $\mathrm{I}$ from the set of condition attributes. $\tau_{R * R_{2}}(X)=$ $\{\phi, U,\{2,4\}\}$

$R_{3}=\{\{1,2,6\},\{3,5\},\{4\}\}$ which is obtained by deleting $R$ from the set of condition attributes. $\tau_{R * R_{3}}(X)=\{\phi, U\}$ $R_{4}(X)=\{\{1,2\},\{1,6\},\{3,5\},\{4,6\}\}$, $\tau_{R * R_{4}}(X)=\{\phi, U,\{4,6\}\} R_{5}(X)=\{\{1,2\},\{1,6\},\{3,5\},\{4\}\}$, $\tau_{R * R_{5}}(X)=\{\phi, U\}$.

Step $4 C_{1}=\{L, I, D, S\}, C_{2}=\{L, I, R, D\}$. Core $=C_{1} \cap C_{2}=$ $\{L, I, D, S\} \cap\{L, I, R, D\}=\{L, I, D\}$. Hence Lump in breast, Inverted nipple and nipple discharge are connected with the breast cancer.

\section{Conclusion}

The rough derived sets and their properties are defined. Theorems on rough interior and rough closure are derived. An algorithm is developed to find the CORE of an information system. Two examples are discussed to find the CORE of an information system. Rough topology concept can be extended for further research 


\section{References}

[1] M.Kryszkiewiecz, Rules in incomplete information systems, Information Sciences, 113(1999), 271-292.

[2] Nirmala Rebecca Paul, Decision making in an information system via a new topology, Annals of Fuzzy Mathematics and Informatics, 9(2016), 1-10.

[3] A.S. Salama, Some topological properties of rough sets with tools for data mining, International Journal of Computer Science, 8(2011), 588-595.

[4] Z.Pawlak, Rough sets, International Journal of Information and Computer Sciences, 11(1982), 341-356.

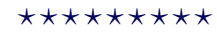

ISSN(P):2319- 3786

Malaya Journal of Matematik

$\operatorname{ISSN}(\mathrm{O}): 2321-5666$

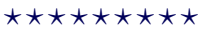

